

\title{
A Statistical Mechanical Description of Biomolecular Hydration
}

\author{
Gerhard Hummer, ${ }^{\dagger}$ Angel E. García and D. Mario Soumpasis* \\ Theoretical Biology and Biophysics Group T-10, MS K710, Los Alamos National Laboratory, Los \\ Alamos, NM 87545, U.S.A
}

We present an efficient and accurate theoretical description of the structural hydration of biological macromolecules. The hydration of molecules of almost arbitrary size (tRNA, antibody-antigen complexes, photosynthetic reaction centre) can be studied in solution and in the crystal environment. The biomolecular structure obtained from x-ray crystallography, NMR, or modeling is required as input information. The structural arrangement of water molecules near a biomolecular surface is represented by the local water density analogous to the corresponding electron density in an x-ray diffraction experiment. The water-density distribution is approximated in terms of twoand three-particle correlation functions of solute atoms with water using a potentials-of-mean-force expansion.

Contribution for: The Royal Society of Chemistry, Faraday Division - Faraday Discussion No. 103 on Hydration Processes in Biological and Macromolecular Systems, Sheffield Hallam University, UK (1-3 April 1996).

\footnotetext{
$\dagger_{\text {also: }}$ Center for Nonlinear Studies

${ }^{*}$ Biocomputation Group, Max Planck Institute for Biophysical Chemistry, P.O. Box 2841, D-37018 Göttingen, Germany
} 


\section{Introduction}

Water forms the natural solution environment of biological macromolecules. It is therefore not surprising that, on a molecular level, water influences many aspects of biological function. ${ }^{1}$ Of foremost importance is the role of water in determining structural equilibria. ${ }^{2}$ Water is a significant factor both in folding and association of macromolecules. A well-known example are so-called hydrophobic interactions. In an aqueous environment, nonpolar atoms and groups preferentially aggregate. Through this aggregation, water can provide a strong driving force in the folding of proteins ${ }^{3}$ and assembly of membranes. In addition, the high dielectric constant of water efficiently screens long-range charge interactions. On shorter length scales, water is a competitor for intramolecular hydrogen bonds. Its unique structure and small size allow water to mediate hydrogen-bond interactions. In macromolecular recognition and association, water can therefore bridge otherwise unsatisfied interaction partners.

In addition to its structural role, water can also directly influence the chemistry of enzymatic reactions such as hydrolysis. Cytochrome P450 molecules can exploit the polar nature of water to adjust redox potentials by changing the amount of internal hydration upon substrate binding. These proteins are highly hydrated in their interior, showing several solvent channels partly connected to the solution. ${ }^{4}$ Particularly well hydrated is the vicinity of the active site, both in the substrate bound and unbound states.

Experimental information about the interactions of water with biomolecules comes predominantly from spectroscopic methods. Nuclear magnetic resonance (NMR) spectroscopy can determine the presence of hydration water molecules with sufficiently long residence times. ${ }^{5} \mathrm{X}$-ray (and neutron ${ }^{6}$ ) spectroscopy of single crystals ${ }^{7}$ (and fibers) give the highestresolution data regarding the structural hydration of biomolecules. Single-crystal diffraction methods can provide a detailed, three-dimensional picture of the localisation of water molecules at the surface and in the interior of biomolecules. But clearly, the application of single-crystal diffraction requires that the biomolecule can be crystallised; and that the crystal diffracts to short enough wavelengths to get the spatial resolution required for unambiguously assigning water molecules. Moreover, re-refinements and studies of crystals with different space groups reveal methodological difficulties in crystallographic hydration 
studies. ${ }^{8,9}$ Surface regions of interest must not be affected by crystal packing but should be exposed to sufficiently large solvent channels in the crystal. X-ray and neutron diffraction crystallography also face problems when the water phase surrounding the biomolecule is disordered. The crystal ordering is much less stringent for small molecules occupying solvent channels, with the consequence that the water structure can vary considerably between different unit cells of the crystal. Overall, this disorder reflects an equilibrium water density distribution in the crystal unit cell. The electron density, as determined in an x-ray diffraction experiment, contains, in principle, the information about this position-dependent one-particle density of water. However, the practical hydration analysis is limited to regions showing high localisation of water molecules.

Theoretical methods are used to overcome some of these obstacles in studying the structural hydration of biomolecules. The current theoretical modeling of biomolecules in solution and in the crystal environment relies almost exclusively on computer simulations. Molecular dynamics simulations (and, used less often, Monte Carlo simulations) of biomolecules and water provide versatile tools to study solute-solvent interactions. ${ }^{10-12}$ These simulations are carried out at the Born-Oppenheimer level, i.e., using classical interactions between atoms. Aside from the difficulties of specifying sufficiently accurate interaction potentials, the major problem associated with computer simulation studies is the enormous computer time requirement. This matters less if a study is devoted to only a single and relatively small molecule. However, the conventional simulation approach rapidly reaches its limitations if a large number of mutations, ${ }^{9}$ large biomolecules such as photosynthetic reaction centres ${ }^{13}$ or antibody-antigen complexes ${ }^{14}$ or a large number of ligands such as in a typical small-drug docking study are analysed.

For large molecules and large sample sizes, fast and accurate methods other than computer simulations are needed. One direction of research is to use so-called "knowledge-based" approaches. ${ }^{15-18}$ Rules are "learned" from a training set of known x-ray crystal structures. These rules are assumed to be transferable to "predict" the hydration of a molecule not in the training set. Despite the interesting results of these approaches, problems remain such as insufficient statistics because of, for instance, the enormous number of side chain 
conformations ${ }^{19}$ and side chain interactions in proteins that prohibit an exhaustive sampling. Another problem is that crystallographic hydration sites often are caused by interactions with two or more symmetry-related molecules in the crystal and are thus not conserved between different crystals. ${ }^{8.9}$

A theory based on molecular principles avoids these difficulties. We shall discuss such a method to describe the equilibrium structural hydration of biomolecules in solution and in the crystal environment. This statistical-mechanical method is based on a truncated expansion of the local density of water in terms of two- and three-particle correlation functions. ${ }^{\dagger} \mathrm{A}$ similar method was used previously to study ionic densities near nucleic acids. ${ }^{21}$ An accurate analysis of the structural hydration even of molecular systems with 10000 or more heavy atoms becomes feasible within CPU minutes, rather than weeks as in comparable computer simulation studies. Unlike "knowledge-based" approaches, the method is soundly based on statistical mechanics. It can be applied to study the hydration of any biomolecular complex without previous knowledge of similar structures.

We shall first develop the theoretical foundation. A formal result for local densities in inhomogeneous systems will be derived. We shall then show how this general theory can be applied to biomolecular systems. Necessary approximations will be emphasised, followed by a discussion of the expected limitations of the formalism. To illustrate the applicability and accuracy of the method, results will be presented for a broad class of systems. Triplet correlations in water will be discussed. Emphasis will be laid on comparison with diffraction experiments and computer simulations. Results will be discussed for the ice-water interface, small peptides ( $\alpha$-helix, $\beta$-hairpin), nucleic-acid fragments and B-DNA oligonucleotides. The structural hydration of transfer RNA, antibody-antigen complexes and a photosynthetic reaction centre will be discussed. Directions for future applications to the calculation of

${ }^{\dagger}$ To bring the study of nonuniform fluids to a more routine status, Percus and Williams ${ }^{20}$ suggest "a technique by which the amassed theory and data of uniform fluids can be suitably inserted into the corresponding nonuniform system." At least in the sense of inserting data, the present method follows this premise. 
binding entropies will be outlined and preliminary results for antibody-antigen complexes will be shown.

\section{Theoretical Development}

\subsection{Local densities in inhomogeneous systems}

Central to hydration calculations is the local water-density distribution, which characterises the structural equilibrium properties of water near a biomolecule. The density distribution is expected to approach the bulk-water density of $1 \mathrm{~g} / \mathrm{cm}^{3}$ within a few angstrom from a biomolecule in solution. Close to the molecular surface, however, the density distribution will show sharp oscillations reflecting the structural ordering of water molecules.

The solvated macromolecule is characterised by atomic coordinates $s_{i_{\alpha}}$ of $N_{\alpha}$ atoms of type $\alpha$ and $M$ different types of atoms, $\alpha=1, \ldots, M$. This set of atomic coordinates, $\left\{\mathrm{s}_{i_{\alpha}}\right\}$, can be obtained from $\mathrm{x}$-ray crystallography, NMR, or modeling. A rigid equilibrium structure will be assumed for the solute. We express the local solvent density as a configuration-space integral. To simplify the presentation, results for an atomic solvent will be given. The generalisation to molecular solvents is straightforward. For a canonical ensemble of $N$ solvent particles with coordinates $\left\{\mathbf{r}_{i}\right\}$, we obtain for the conditional solvent density at a position $\mathbf{r}_{1}$

$$
\rho\left(\mathbf{r}_{1} \mid\left\{\mathbf{s}_{i_{\alpha}}\right\}\right)=N \frac{\int d \mathbf{r}_{2} \cdots d \mathbf{r}_{N} \exp \left[-\beta U\left(\left\{\mathbf{r}_{i}\right\},\left\{\mathbf{s}_{i_{\alpha}}\right\}\right)\right]}{\int d\left\{\mathbf{r}_{i}\right\} \exp \left[-\beta U\left(\left\{\mathbf{r}_{i}\right\},\left\{\mathbf{s}_{i_{\alpha}}\right\}\right)\right]},
$$

where $\beta=1 / k_{\mathrm{B}} T$ and $U$ is the total potential energy in a classical mechanical description. In a computer simulation study, eqn (1) is used directly to obtain local water densities as ensemble or, equivalently, time averages. Here, we further simplify eqn (1). The first step is to express the local density in terms of particle correlation functions, ${ }^{22,23}$

$$
\rho\left(\mathbf{r} \mid\left\{\mathbf{s}_{i_{\alpha}}\right\}\right)=\rho_{0} \frac{g^{\left(1 ;\left\{N_{\alpha}\right\}\right)}\left(\mathbf{r},\left\{\mathbf{s}_{i_{\alpha}}\right\}\right)}{g^{\left(\left\{N_{\alpha}\right\}\right)}\left(\left\{\mathbf{s}_{i_{\alpha}}\right\}\right)},
$$

where $\rho_{0}=N / V$ is the solvent density in the volume $V$. Eqn (2) follows directly from the definition of multiparticle correlation functions $g^{(n)}$. However, eqn (2) also shows a close analogy with probability theory relating the conditional probability of observing event 
$A$ given event $B$ to the ratio of the probability of event $A$ and $B$ to that of event $B$, $P(A \mid B)=P(A \cap B) / P(B)$. The high dimensionality of the correlations in eqn (2) prohibits a direct application. We therefore apply a potential-of-mean-force (PMF) expansion. ${ }^{22,24,25}$ This yields an expression for the density in terms of lower-order correlations, ${ }^{23,26}$

$$
\begin{aligned}
& \rho\left(\mathbf{r} \mid\left\{\mathbf{s}_{i_{\alpha}}\right\}\right)=\rho_{0}\left[\prod_{\alpha=1}^{M} \prod_{i_{\alpha}=1}^{N_{\alpha}} g^{(1 ; \alpha)}\left(\mathbf{r}, \mathbf{s}_{i_{\alpha}}\right)\right] \\
& \times\left[\prod_{\alpha=1}^{M} \prod_{j_{\alpha}=1}^{N_{\alpha}} \prod_{\beta=\alpha}^{M} \prod_{j_{\beta}=1+\delta_{\alpha \beta} j_{\alpha}}^{N_{\beta}} \frac{g^{(1 ; \alpha, \beta)}\left(\mathbf{r}, \mathbf{s}_{j_{\alpha}}, \mathbf{r}_{j_{\beta}}\right)}{g^{(1 ; \alpha)}\left(\mathbf{r}, \mathbf{s}_{j_{\alpha}}\right) g^{(\alpha, \beta)}\left(\mathbf{s}_{j_{\alpha}}, \mathbf{s}_{j_{\beta}}\right) g^{(\beta ; 1)}\left(\mathbf{s}_{j_{\beta}}, \mathbf{r}\right)}\right] \cdots
\end{aligned}
$$

where $\delta_{\alpha \beta}$ is the Kronecker symbol. To lowest order, the expansion gives the bulk density of water, $\rho_{0}$. The second- and third-order corrections involve products over all single solute atoms and all distinct pairs, respectively. Truncation at the pair- and three-particle correlation level corresponds to the well-known Kirkwood ${ }^{24}$ and Fisher-Kopeliovich ${ }^{27}$ superposition approximations, KSA and FKSA, respectively.

\subsection{Towards a description of the structural hydration of biomolecules}

Eqn (3) involves a prohibitively large number of correlation functions when applied to a macromolecule in aqueous solution. However, based on energetic and geometric arguments it is possible to group atoms into similar classes. This reduces drastically the number of different atom types required in the calculations. Hydrogen bonds provide the strongest interactions between water and a biomolecule. In proteins and nucleic acids, the electronegative oxygen, nitrogen and, to a smaller extent, sulfur atoms are possible interaction partners for water to form hydrogen bonds. Geometrically, these hydrogen bonds are similar (in bond length and bond angle) to those formed between water molecules.

This observation allows us to obtain a description of the structural hydration that should capture the essential features by equating all nitrogen, oxygen and sulfur atoms to water oxygen with respect to their effect on ordering water. Nonpolar carbon atoms are expected to have only a local, weakly structuring effect on water. ${ }^{28,29}$ Here, we shall represent carbons as excluded volume by setting the water-oxygen density to zero inside a sphere with radius $r_{c}=0.3 \mathrm{~nm}$, where $r_{c}$ corresponds approximately to the distance of closest approach of 
a water oxygen to a methane in aqueous solution. The carbon-water pair correlations in eqn (3) are thus represented by a unit step function, $\Theta\left(r-r_{c}\right)$. Three-particle correlations involving carbon atoms are neglected. If large hydrophobic regions are exposed to water, the treatment needs to be refined as discussed below. ${ }^{29}$ The solvent-exposed surface of proteins and nucleic acids is mostly hydrophilic. Hydration calculations based on just polar atoms should therefore prove to be sufficiently accurate.

Another possible complication arises from the treatment of charged groups. One could generate a more extensive data base of correlation functions for different charged groups with water. However, in nucleic acids and proteins, the charges on charged groups reside essentially on oxygens and nitrogens as well as on polar hydrogens attached to them. The characteristics of the structural hydration of charged groups are therefore expected to be represented well by considering just oxygen and nitrogen atoms and, possibly, polar hydrogens.

Polar hydrogens on non-rotating groups such as $\mathrm{NH}$ and $\mathrm{NH}_{2}$ can be treated explicitly to better represent the directionality of hydrogen bonds. A correlation function $\chi\left(\mathbf{r} \mid \mathbf{s}_{D}, \mathbf{s}_{H}\right)=$ $\rho\left(\mathbf{r} \mid \mathbf{s}_{D}, \mathbf{s}_{H}\right) / \rho_{0} g^{(2)}\left(\mathbf{r}, \mathbf{s}_{D}\right)$ describes the conditional water-oxygen density caused by an $\mathrm{O}-\mathrm{H}$ hydrogen-bond donor with $\mathrm{O}$ at $\mathrm{s}_{D}$ and $\mathrm{H}$ at $\mathrm{s}_{H}$. We thus obtain a working formula,

$$
\begin{aligned}
\rho\left(\mathbf{r} \mid \mathbf{s}_{1}, \ldots, \mathbf{s}_{n}\right)= & \rho_{0} \prod_{i=1}^{n} g^{(2)}\left(\mathbf{r}, \mathbf{s}_{i}\right) \prod_{j=1}^{n-1} \prod_{k=j+1}^{n} \frac{g^{(3)}\left(\mathbf{r}, \mathbf{s}_{j}, \mathbf{s}_{k}\right)}{g^{(2)}\left(\mathbf{r}, \mathbf{s}_{j}\right) g^{(2)}\left(\mathbf{s}_{j}, \mathbf{s}_{k}\right) g^{(2)}\left(\mathbf{s}_{k}, \mathbf{r}\right)} \\
& \times \prod_{\text {carbons } \mathbf{s}_{C}} \Theta\left(\left|\mathbf{r}-\mathbf{s}_{C}\right|-r_{c}\right) \prod_{\substack{\text { polar hydrogens } \\
\left.\text { (with donors } \mathbf{s}_{D}\right)}} \chi\left(\mathbf{r} \mid \mathbf{s}_{D}, \mathbf{s}_{H}\right),
\end{aligned}
$$

$g^{(2)}$ and $g^{(3)}$ are the two- and three-particle correlation functions of water oxygens in bulk water, respectively; $s_{1}, \ldots, s_{n}$ are the positions of electronegative atoms. Unless otherwise stated, the correction for polar hydrogens, $\chi$, was not applied in the following calculations.

\subsection{Expected limitations}

A series of approximations was made in the development of the PMF method for biomolecular hydration calculations. The first and most prominent is the truncation of the expansion eqn (3) at the three-particle correlation level. Truncation at the two-particle level corresponds to using the KSA, which was studied extensively as an approximation for 
three-particle correlations. For simple liquids with spherical, pair-additive interaction potentials, explicit computer simulation comparisons with three-particle correlations showed that structurally the KSA approximation works reasonably well, with the largest errors at particle contact. ${ }^{30-32}$ This view was supported by theoretical studies of three-particle correlations based on diagrammatic expansions and integral equations. ${ }^{30,31,33,34}$ However, it was also demonstrated that the KSA as a closure of the Yvon-Born-Green hierarchy gives far less satisfactory results. ${ }^{35}$

For a molecular fluid such as water with a highly anisotropic interaction potential owing to the directional hydrogen-bond interactions, one expects the KSA to give very poor structural results at distances corresponding to the first hydration layer. This is best understood if one looks at the structure of hexagonal ice, where a water molecule has only four nearest neighbours in a tetrahedral arrangement. Water-oxygens in contact form isosceles triangles with edges of about $0.28,0.28$ and $0.45 \mathrm{~nm}$ length. Locally ice-like structures will also be observed in liquid water. Equilateral triangles with edges of $0.28 \mathrm{~nm}$ length would result in strongly unfavourable interactions. Since the hydrogen-bond interactions dominate under standard conditions of temperature and density, water is not well-packed and the KSA gives poor results for three-particle correlations. Therefore, three-particle correlations are indispensable.

It is less clear, how well the FKSA approximates four-particle correlations. Computersimulation calculations of four-particle correlations are not trivial because of insufficient statistical sampling. We can instead identify the smallest tetrahedron (as measured by its longest edge) in an ice lattice which would be given a finite FKSA probability, but does not appear in the lattice. In hexagonal ice, the smallest three triangles formed by oxygen atoms on lattice sites have edges $A=(1,1, \sqrt{24} / 3), B=(1, \sqrt{24} / 3,5 / 3)$ and $C=$ $(\sqrt{24} / 3, \sqrt{24} / 3, \sqrt{24} / 3)$ in units of the nearest-neighbour distance (ca. $0.28 \mathrm{~nm}$ ). Among all possible combinations of triangles $A, B$ and $C$ in Euclidian space, a combination of three triangles $\mathrm{B}$ can be formed into a tetrahedron that is not found in the lattice. Therefore, the smallest quadruplet in hexagonal ice incorrectly predicted by the FKSA is rather large having four of its six edges longer than $0.45 \mathrm{~nm}$. Its contributions should therefore be relatively 
small. More convincing, however, than these lattice-based arguments, are the results of the following section comparing calculations based on the FKSA for density profiles at an idealised ice-water interface with explicit computer simulations.

Another problem associated with the PMF expansion for correlation functions is its multiplicative form. As a consequence, the expansion expressions for local densities, eqns (3) and (4), are highly nonlinear in the correlation functions. This problem will surface when one attempts to use eqn (3) with correlation functions for all atoms. For any given point at the molecular surface, a large number of factors will then contribute to the calculated density. The use of only polar atoms (with nonpolar atoms modeled as excluded volume) has therefore the advantage that statistical errors in the correlation functions and systematic errors in the truncated expansion expression are better controlled because the number of contributing terms is relatively small.

In applications to biomolecular hydration calculations, the first problem arises from the assumption of a rigid equilibrium structure. As a consequence, correlated fluctuations of water and surface residues of the hydrated biomolecule are neglected. This problem is expected to be less significant if the biomolecule has a well defined structure. If however large motions occur at the biomolecular surface, only a truly dynamic study is expected to describe well these nonlinear fluctuations.

Regarding the effect of hydrophobic residues on the local water density in their vicinity, recent calculations ${ }^{29}$ showed that a PMF expansion is satisfactory if individual nonpolar groups do not overlap. For covalently bonded hydrocarbons, however, serious deficiencies of a PMF expansion for nonpolar molecules become apparent. The strongest interaction of nonpolar groups with water is volume exclusion. If individual sites overlap, each describing a spherical excluded volume, a truncated product expansion eqn (3) guarantees density zero in the interior but for the exterior the predicted density will be wrong. The worst case is the limit of complete overlap of $n$ hard spheres, where the FKSA, eqn (3), results in a density profile $\rho_{0}[g(r)]^{n-n(n-1) / 2}$ compared to the correct result of $\rho_{0} g(r)$ where $g(r)$ is the hard sphere-water pair correlation function. ${ }^{29}$ However, the effect of nonpolar solutes in water can be described well by the simplest approximation based on proximity, i.e., taking only a 
pair correlation with the nearest solute site. ${ }^{28,29}$

As a consequence of the discussed approximations and limitations, one can expect good qualitative results for the PMF expansion of the density. This means that the PMF expansion should primarily be able to locate regions with high local water density at a molecular surface. However, the actual density values are less reliable. The analysis of the structural hydration using PMF calculations should therefore primarily focus on the identification of regions with high water density.

\section{Results and discussion}

\subsection{Water correlation functions}

Pair correlations of water were studied extensively by $\mathrm{x}$-ray and neutron diffraction experiments $^{36,37}$ and computer simulations using various water models. ${ }^{38,39}$ Much less is known about water triplet correlations. Experimentally, water three-particle correlations have been accessible only through indirect, maximum-entropy type approaches. ${ }^{40}$ Using computer simulations, water three-particle correlations were first calculated by Soumpasis et $a{ }^{41}$ for the $a b$ initio $\mathrm{NCC}^{42}$ water model. In Fig. 1, we show results for the three-particle correction, $\Gamma(r, r, s)=g^{(3)}(r, r, s) /\left[g^{(2)}(r)\right]^{2} g^{(2)}(s)$, of the SPC model of water ${ }^{43}$ for isosceles (and equilateral) triangles with edges $r, r$ and $s$ formed by three water oxygens. The simulation calculations were described previously. ${ }^{22,23}$ For oxygen pair distances between 0.19 and $0.25 \mathrm{~nm}$, a constrained method was used to determine $g^{(3)} .{ }^{23}$ Unless otherwise stated, SPC correlations will be used in the PMF calculations.

As expected from our previous discussion based on the structure of hexagonal ice, the locally tetrahedral organisation of water results in $\Gamma$ being close to zero for three oxygens in hydrogen-bond distance $0.28 \mathrm{~nm}$. For isosceles triangles with distances close to the ice-values for nearest neighbours, $0.28,0.28$ and $0.45 \mathrm{~nm}, \Gamma$ reaches its maximum for isosceles triangles. In addition, Fig. 1 shows that the triplet correction is of short range, decaying rapidly to one. For equilateral triangles with $r>0.3 \mathrm{~nm}, \Gamma$ deviates less than $20 \%$ from 1 . These results have important consequences for the PMF density expansion: Triplet corrections are indispensable at short range, but contribute little at larger distances. 


\subsection{A test case: The interface of ice and water}

To illustrate the applicability of the expansion formula eqn (3) for inhomogeneous aqueous systems, we study a model ice-water interface. The interface is described by fixing SPC water oxygens at ideal hexagonal-ice Ih lattice positions without specifying molecular orientations. The thermal disorder in the solid phase is limited to reorientation of water molecules. The basal plane of the ice layer is covered by liquid water. More realistic models of the interface were studied previously by computer simulations. ${ }^{44-47}$

Fig. 2 shows results of explicit Monte Carlo computer simulations and PMF calculations for the density profile as a function of the distance $d$ from the plane defined by the last layer of ice-oxygen atoms. ${ }^{22}$ In the PMF expansion, the discrete two- and three-particle correlation functions of SPC water are used with linear and trilinear interpolation for pair distances. The PMF expansion truncated at the two-particle level clearly fails to reproduce the simulation densities. The first peak is too close because of the high KSA probability of equilateral triangles. The second peak at the KSA level is too small. If, however, threeparticle correlations are included, the picture improves drastically. Position and height of the first peak are well reproduced by the FKSA-PMF density. Beyond the first peak, qualitative agreement is observed. The FKSA-PMF result is less structured, but shows extrema at the right positions. In biomolecular applications, the emphasis is on identifying high-density regions. Therefore, accuracy of the FKSA-PMF expansion in the interfacial region is important, where the strongest variations in the density occur.

\subsection{Local density of water around rigid peptides}

To test the accuracy of the PMF method for biomolecular hydration, we performed molecular dynamics (MD) simulations of an $\alpha$-helix and a $\beta$-hairpin in aqueous solution. The local density of water around each polypeptide is calculated by averaging occupancy numbers

of water molecules in a uniformly spaced three-dimensional grid. Constant $(N, V, T=$ $300 \mathrm{~K}$ ) simulations have been extended for $1.0 \mathrm{~ns}$ with a time step of $0.002 \mathrm{ps}$, after an equilibration period of $125 \mathrm{ps}$. The united-atom OPLS force field and the rigid TIP3P model of water ${ }^{48}$ were used for all simulations. $\mathrm{N}$ - and $\mathrm{C}$-terminal residues were uncharged. The 
PMF calculations used eqn (4), including the hydrogen-bond correction $\chi$. The correlation functions $g^{(2)}, g^{(3)}$ and $\chi$ were those of TIP3P water.

A polypeptide, $\mathrm{Ala}_{18}$, was constructed in an ideal $\alpha$-helical conformation with $\phi=-48^{\circ}$, $\psi=-57^{\circ}$. As a system that better represents the composition of amino acids in a protein, we have chosen a $\beta$-hairpin from BPTI with sequence ${ }^{21} Y F Y N A K A G L C Q T^{32}$. The conformation of this peptide was taken from the crystal structure of Wlodawer et al. ${ }^{49}\left(\mathrm{PDB}^{50}\right.$ code 5pti). This peptide contains one charged amino acid (K26), polar residues (Y21, Y23, N24, Q31 and T32), a cysteine residue (C30), four non-polar residues (F22, A25, G26 and L27) and three residues with aromatic rings (F22, Y21 and Y23). In BPTI, C30 forms a disulfide bridge with $\mathrm{C} 51$. Here, we take $\mathrm{C} 30$ in the reduced form.

The peptides were immersed in a previously equilibrated cubic box of water. All water molecules in this box that were within a distance of $0.27 \mathrm{~nm}$ to a non-hydrogen atom of the peptide were deleted from the system. The resulting $\alpha$-helix ( $\beta$-hairpin) system contains 112 (125) peptide atoms - including united atoms and hydrogen atoms forming part of polar groups - and 266 (255) water molecules. The $\beta$-hairpin carries a net charge of +1 . The peptides were kept rigid during the simulations. The Coulomb interactions were modeled by a generalised reaction field (GRF) ${ }^{22}$ with a cutoff of $0.95 \mathrm{~nm}$. The local water density was calculated in a cubic grid with ca. $0.1 \mathrm{~nm}$ width and averaged over 10000 configurations. The local density of water gives values as high as $15 \rho_{0}$ ( $\alpha$-helix) and $16 \rho_{0}$ ( $\beta$-hairpin). Block-averaged local densities show a uniform error in the average of $\pm 2 \rho_{0}$ for densities over $3 \rho_{0}$.

Fig. 3(a) shows the local water density around the ideal $\alpha$-helix. The local water densities calculated by both MD and PMF calculations are in good agreement. A region where the density does not compare well is the C-terminus, with PMF overestimating the extent of the high-density region. Fig. 3(b) shows several views of the hydration pattern of the $\beta$-hairpin. There is excellent agreement between the MD and PMF densities at the $\mathrm{NH}_{3}^{+}$of Lys, the SH group of Cys (with $\mathrm{S}$ coloured in yellow) and various $\mathrm{NH}$ (with $\mathrm{N}$ coloured in blue), $\mathrm{OH}$ and $\mathrm{CO}$ (with $\mathrm{O}$ coloured in red) groups in the peptide. We find that the SH group in Cys is well represented by the water correlation functions and, therefore, SH should 
be treated as a polar group. There are two regions where the PMF and MD calculated densities do not agree. The MD simulation show large density of water (5.0) at a site surrounded by a carbonyl oxygen ( $\mathrm{O}$ of $\mathrm{C} 30$ at hydrogen-bond distance of $0.263 \mathrm{~nm}$ ) and five carbon atoms (CB, CG, CD1, CD2 of L29 and CG of N31) at distances close to contact $(0.375,0.423,0.403,0.442$ and $0.413 \mathrm{~nm}$, respectively). The MD simulation shows another region of high density (4.0) at a site surrounded by four polar groups beyond hydrogen-bond distance $(0.38$ to $0.45 \mathrm{~nm})$ and near contact with CB of T32 $(0.368 \mathrm{~nm})$ and close to the N-terminal acetyl methyl $(4.10 \mathrm{~nm}$ ), CG2 of T32 and CA of G28 (at 0.423 and $0.427 \mathrm{~nm}$, respectively). In summary, PMF and simulation results for the positions of high density agree well. Significant discrepancies are observed only when water molecules are localised by interactions with several nonpolar groups and only one or two polar groups. For these cases, a refined treatment of combined polar and nonpolar groups is required, as outlined above.

\subsection{Crystals of small nucleic-acid molecules}

Crystals of small nucleic-acid fragments were studied in ref. 26. The structures of these small-molecule crystals were determined with high x-ray crystallographic resolution, such that the positions of ordered water molecules could be determined with high accuracy. With one exception, the solvent channels in the crystals were small such that most of the water molecules were well ordered. The PMF hydration calculations showed spatial regions of high water density $\left(\geq 5 \rho_{0}\right)$ in excellent agreement with crystal-water positions. In spatially extended solvent channels, PMF high-density regions connect different crystal-water sites, indicative of flexibility in the hydration network. This also agrees with the crystallographic observations: Seeman et al. ${ }^{51}$ proposed to view the hydration of these crystals on the basis of a solid-liquid interface with thermal and statistical disorder in the water phase, to better account for the diffraction data than a conventional model using Gaussian electron-density distributions for water sites.

The radial dependence of the calculated water density around crystal water sites was peaked at or near $r=0$ for most of the crystal-water positions. This demonstrates good 
positional agreement of calculated and measured water positions. However, the agreement was less satisfactory for integrated densities. When the integration extended over the volume of approximately one water molecule, sites with highly peaked densities contained less than one water molecule (typically 0.3 to 0.8 ), whereas less ordered regions often showed integrated densities closer to one. This suggests that in the PMF calculations, water-density peaks might actually be underestimated, although positioned correctly.

\subsection{B-DNA oligomers}

The hydration of B-DNA oligomers has been studied extensively using a variety of experimental techniques ${ }^{52,53}$. In X-ray crystallographic and, more recently, NMR studies, ${ }^{54,55}$ the minor groove showed the most conserved hydration patterns. Two characteristic features emerged: In A.T base-pair regions, a highly-localised so-called spine of hydration ${ }^{56,57}$ occupies the minor groove. Two less localised (and less conserved) side-by-side ribbons of high water density ${ }^{58,59}$ are found in wider G.C base-pair regions of the minor groove, Interestingly, a re-refinement of the dodecamer d(CGCGAATTCGCG) $)^{56,57}$ by Westhof ${ }^{60}$ showed that the second layer of the water spine was less localised. Lower occupancies were assigned and the positions slightly shifted with respect to the original refinement.

PMF calculations of B-DNA oligomer hydration did reproduce these experimental findings. ${ }^{23,61,62}$ An interesting finding of those PMF studies was that hydration in the minor groove shows cooperativity. To account for the influence of neighbouring bases, the structural hydration in the minor groove is best described as a function of base-pair steps. In particular, marked differences were observed for the hydration of $5^{\prime}-\mathrm{TA}-3^{\prime}$ steps compared to $5^{\prime}$-AT- $3^{\prime}$ steps with TA steps showing less water localisation and disruption of the spine of hydration. ${ }^{23,61,62}$ This is in agreement with earlier findings based on simple energetic arguments for the interaction of a single water molecule with $\mathrm{DNA}^{63}$ and later NMR hydration studies comparing AATT with TTAA sequences. ${ }^{64}$ 


\subsection{Transfer RNA molecules}

Crystallisation and $x$-ray structure determination of transfer RNA (tRNA) molecules ${ }^{65}$ made it possible to study tRNA hydration. ${ }^{66}$ Westhof et al.$^{8,66}$ discussed results for a small region in tRNA Asp involving bases A9, G10 and G45. Water molecules in that region appear to be conserved between $\mathrm{tRNA}^{\mathrm{Asp}}$ and $\mathrm{tRNA}^{\text {Phe }}$. However, the assignment of water is difficult as the crystallographic resolution of these structures is only $0.27 \mathrm{~nm}\left(\mathrm{tRNA}^{\mathrm{Phe}}\right.$ ) and $0.3 \mathrm{~nm}\left(\mathrm{tRNA}^{\mathrm{Asp}}\right)$.

Results of a PMF calculation for that region are shown in Fig. 4. We compare calculations for two structures $\mathrm{A}$ and $\mathrm{B}$ of tRNA ${ }^{\mathrm{Asp}}$ with PDB codes 2 tra and $3 \operatorname{tra}^{67}$ as well as a tRNA ${ }^{\text {Phe }}$ structure $\left(6 \operatorname{tna}^{65}\right)$, which was later re-refined by Westhof et al. $\left(4 \operatorname{tra}^{67}\right)$. The calculations for tRNA ${ }^{\text {Asp }}$ were performed both for isolated molecules and in the crystal environment to identify effects of crystal contacts.

The PMF hydration calculations indeed reveal some degree of conservation between different crystals and different tRNA molecules in the region of bases 9,10 and 25 forming the interface between the dihydro-uridine loop and the anti-codon stem. ${ }^{66}$ The conserved water molecules identified by Westhof $e t$ al. appear at or close to regions with high calculated water density. In particular, although none of the water molecules could be assigned in 2 tra, the calculated high-density regions agree well with those of 3tra. PMF calculations indicate that one of the water molecules in 2tra and 3tra (marked with a green arrow in Fig. 4) can in part be attributed to crystal packing. The PMF calculations show only few grid points with density between 3 and $4 \rho_{0}$ near that water site for the isolated molecules, but densities of $7 \rho_{0}$ in the crystal.

PMF calculations can therefore provide direct information about the effects of crystal packing on the hydration structure. In addition, PMF calculations allow to analyse the hydration even for structures with intermediate to low resolutions, where the crystallographic assignment of water is difficult. 


\subsection{Antibody-antigen complexes}

The $x$-ray structure of a complex of the Fv fragment of the anti-hen egg white lysozyme (HEL) antibody D1.3 with its antigen bound was solved at $0.18 \mathrm{~nm}$ resolution (PDB code $\left.1 \mathrm{vfb}^{14}\right)$. An earlier study of the larger Fab fragment of the D1.3 antibody complexed to HEL was solved at a lower resolution of $0.25 \mathrm{~nm}$ (PDB code $1 \mathrm{fdl} \mathrm{l}^{68}$ ). In Fig. 5, results of PMF calculations are shown for the antibody-antigen contact regions of the $1 \mathrm{vfb}$ and 1fdl structures. Only the higher-resolution 1vfb crystal structure (as deposited at PDB) contains water positions, and only in the contact interface. Remarkably, the interface is strongly hydrated. In $1 \mathrm{vfb}$, water molecules were found in partly connected clusters in the interface, thus correcting for a lack of direct surface complementarity of antigen and antibody.

The crystal-water sites are, with few exceptions, within or near high water-density regions of the PMF calculations (Fig. 5). The reverse holds as well: In or near regions of high calculated density, one also finds crystal water (no water molecules were reported outside the interface, however). For the lower-resolution $1 \mathrm{fdl}$ structure, the predicted hydration agrees almost perfectly with that of $1 \mathrm{vfb}$.

This illustrates that PMF calculations are rather insensitive to small structural changes, such as those between two crystal structures with different resolution. The agreement with the crystallographic hydration structure shows that PMF calculations can indeed be used in cases where unambiguous assignment of water is difficult, as for the Fab-D1.3 complex 1fdl. And PMF calculations can complement or substitute crystallographic hydration studies when only a low-resolution structure is available and water cannot be assigned unambiguously.

\subsection{Photosynthetic reaction centre}

Deisenhofer $e t a l .^{13}$ presented an x-ray structure of the photosynthetic reaction centre at a high resolution of $0.23 \mathrm{~nm}$ resolution (PDB code 1prc). Strongly hydrated interior regions were identified near the special pair and near the ubiquinone $Q_{B}$. In Fig. 6 , we analyse those regions using PMF calculations. Again, we find agreement between the crystal-water 
positions and regions of high calculated density. Interestingly, the water hydrating the special pair and its nearest haem appear as highly localised PMF density peaks. The hydration structure near the ubiquinone on the other hand shows wires of high density connecting individual water sites. Deisenhofer et al. ${ }^{13}$ suggested that these water molecules near the ubiquinone $Q_{B}$ could play a role in the protonation of $Q_{B}$. The network-type structure observed in the PMF calculations indicates flexibility of the water structure in that region. Mobility is important for water to perform its function as a polar molecule screening charge interactions and lowering electrostatic barriers. Thus, the results of the PMF hydration calculation are further indications of a possible functional role of those water molecules near $\mathrm{Q}_{B}$.

\subsection{Future directions: Energetics of antibody-antigen binding}

The crystallographic and PMF analysis of the antibody D1.3 complexed to its antigen lysozyme revealed a strongly hydrated interface. Thermodynamic data ${ }^{14}$ show that the binding is accompanied by a significant negative entropy at room temperature. For a series of anti-lysozyme antibodies, we tried to correlate the degree of water delocalisation upon binding with the measured binding entropies. As measures for the delocalisation of water, we used the lowest-order term in an entropy expansion in terms of correlation functions, ${ }^{69}$

$$
\sigma=-k_{B} \int d \mathbf{r} \rho(\mathbf{r}) \ln \left[\rho(\mathbf{r}) / \rho_{0}\right] .
$$

The change of $\sigma$ upon binding, $A+B \rightarrow A B$, is $\Delta \sigma=\sigma_{A+B}-\sigma_{A}-\sigma_{B}$. The PDB structure of the complex $A B$ is used. The isolated molecules are those of the complex with $B$ or $A$ removed. Clearly, this treatment is very crude. It assumes that entropies associated with orientational degrees of freedom of water, side chain orientations and higher-order correlations cancel each other in $\Delta \sigma$ or are constant for a class of antibodies. Therefore, we can at the best expect to get the trends right in a comparison with actual measurements. However, $\Delta \sigma$ should describe approximately the entropic effects of changes in polar hydration upon binding.

Fig. 7 shows results for six complexes of antibodies with lysozyme: D1.3 (1vfb ${ }^{14}$ and $\left.1 \mathrm{fdl}{ }^{68}\right)$, D11.15 $\left(1 \mathrm{jhl}^{70}\right)$, D44.1 ( $1 \mathrm{mlc}^{71}$ with two complexes in the asymmetric unit), F9.13.7 
$\left(1 \mathrm{fbi}^{72}\right)$, HyHel-5 $\left(3 \mathrm{hfl}^{73}\right)$ and HyHel-10 $\left(3 \mathrm{hfm}^{74}\right)$. We indeed find a positive correlation between measured entropy and calculated $\Delta \sigma$. Also, calculations always show positive entropies of complex formation. The major neglected contribution is loss of side-chain orientational degrees of freedom upon binding, which would make entropy estimates more negative. The calculated absolute values are of the right order of magnitude, but the variation of experimental values between different antibodies is about twice that of $\Delta \sigma$.

Comparison of the results for antibodies with more than one crystal structure (D1.3) or more than one complex in the asymmetric unit (D44.1 and F9.13.7) show quite a wide spread of $\Delta \sigma$. In the case of D1.3, this spread is mostly caused by differences in the hydration of the individual molecules and not the complex. An improved modeling could therefore use the crystal structures of individual molecules, if available.

We also used a different measure for the water delocalisation,

$$
\omega\left(\rho_{c}\right)=\rho_{0} \int d \mathbf{r} \Theta\left[\rho(\mathbf{r})-\rho_{c}\right],
$$

measuring the extent of regions with density above $\rho_{c}$. For a density threshold $\rho_{c}=3 \rho_{0}$, we observed a somewhat better correlation between $\Delta \omega$ and measured entropies compared to that of $\Delta \sigma$.

It is clear that this kind of entropic analysis is only at a preliminary stage. Antibodyantigen complexes are sufficiently complicated that no final conclusions can be drawn. Conformational searches in ligand-docking should prove to be more accessible to this kind of analysis because there the changes are more local and trends should be easier to see. A simple example is a small molecule replacing one or a few well-localised water molecules. The enthalpic contributions of this binding reaction can be estimated using conventional force fields. $\Delta \sigma$ should give a rough estimate of the entropic contributions owing to dehydration which is otherwise difficult to calculate.

\section{Conclusions}

We have presented a method to study theoretically the structural hydration of biomolecules. The local density of water near a macromolecule in solution or in a crystal is 
expressed in terms of particle correlation functions. These correlations have to be calculated only once and can then be used for any number of molecules. This results in a computationally very efficient method to study the structural hydration of large biomolecules. Illustrating this point, the PMF calculation for an antibody-antigen complex (volume $4.2 \times 4.2 \times 2.1 \mathrm{~nm}^{3}$; $1.4 \times 10^{6}$ grid points) took less than 25 CPU minutes on a Silicon Graphics Indigo 2 workstation. Computer simulations of comparable systems ${ }^{75}$ require significantly larger resources. In addition, a study of the hydration of the interfacial region is difficult using computer simulations, as the diffusion of water molecules into and out of interfacial cavities will occur on much longer time scales as currently accessible to molecular dynamics. Therefore, in that most interesting region, equilibrium averages cannot be calculated easily.

We have shown that PMF calculations show good agreement with computer simulations and crystallographic studies. However, it is important to notice that none of the methods to study structural hydration is without limitations. Crystallographic studies require structures at high resolution with no crystal contacts in the region of interest. In simulations, model potentials have to be specified and even moderate statistical sampling of water densities on coarse grids requires long simulation times. PMF calculations rely on several approximations, but avoid the problem of insufficient statistics. In addition, as individual contributions can be identified and structural variations can be considered easily, PMF calculations can provide real insight.

PMF calculations can be used to study the hydration in the interior and at the surface of biological macromolecules in solution and in the crystal environment. Potential water sites can be identified on the basis of the calculated water-density distributions. Effects of crystal packing on the hydration structure can be estimated. Hydration studies of NMR and intermediate-to-low resolution $x$-ray structures are possible, where experimental information on hydration is limited. Beyond assigning water positions, the equilibrium density distribution contains information about the mobility of water. For instance, the water molecules hydrating the ubiquinone $Q_{B}$ in the reaction centre $1 \mathrm{prc}^{13}$ are connected through wires of high density, indicating flexibility in the hydration structure. Additional applications are the interactive modeling of structurally changed or mutated molecules, where no experimen- 
tal data are available, ${ }^{4}$ or the screening of large numbers of structures in a small-molecule docking study.

\section{References}

1 J. A. Rupley and G. Careri, Adv. Protein Chem., 1991, 41, 37.

2 G. Némethy, W. J. Peer and H. A. Scheraga, Annu. Rev. Biophys. Bioeng., 1981, 10, 459.

3 K. A. Dill, Biochem., 1990, 29, 7133.

4 T. I. Oprea, G. Hummer and A. E. García, submitted, 1995.

5 G. Otting, E. Liepinsh and K. Wüthrich, Science, 1991, 254, 974.

6 P. Langan, V. T. Forsyth, A. Mahendrasingam, W. J. Pigram, S. A. Mason and W. Fuller, J. Biomol. Struct. Dyn., 1992, 10, 489.

7 H. Savage and A. Wlodawer, Methods in Enzymol., 1986, 127, 162.

8 E. Westhof, Annu. Rev. Biophys. Biophys. Chem., 1988, 17, 125.

9 X.-J. Zhang and B. W. Matthews, Protein Science, 1994, 3, 1031.

10 C. L. Brooks III and M. Karplus, Methods in Enzymol., 1986, 127, 369.

11 M. Gerstein and R. M. Lynden-Bell, J. Phys. Chem., 1993, 97, 2982.

12 R. M. Brunne, E. Liepinsh, G. Otting, K. Wüthrich and W. F. van Gunsteren, J. Mol. Biol., 1993, 231, 1040.

13 J. Deisenhofer, O. Epp, I. Sinning and H. Michel, J. Mol. Biol., 1995, 246, 429.

14 T. N. Bhat, G. A. Bentley, G. Boulot, M. I. Greene, D. Tello, W. Dall'Acqua, H. Souchon, F. P. Schwarz, R. A. Mariuzza and R. J. Poljak, Proc. Natl. Acad. Sci. USA, 1994, 91, 1089. 
15 N. Thanki, Y. Umrania, J. M. Thornton and J. M. Goodfellow, J. Mol. Biol., 1991, 221, 669.

16 W. R. Pitt, J. Murray-Rust and J. Goodfellow, J. Comp. Chem., 1993, 14, 1007.

17 B. Schneider, D. M. Cohen, L. Schleifer, A. R. Srinivasan, W. K. Olson and H. M. Berman, Biophys. J., 1993, 65, 2291.

18 S. M. Roe and M. M. Teeter, J. Mol. Biol., 1993, 229, 419.

19 R. L. Dunbrack Jr. and M. Karplus, J. Mol. Biol., 1993, 230, 543.

20 J. K. Percus and G. O. Williams, J. Chem. Phys., 1983, 79, 3009.

21 R. Klement, D. M. Soumpasis and T. M. Jovin, Proc. Natl. Acad. Sci. USA, 1991, 88, 4631.

22 G. Hummer and D. M. Soumpasis, Phys. Rev. E, 1994, 49, 591.

23 G. Hummer and D. M. Soumpasis, Phys. Rev. E, 1994, 50, 5085.

24 J. G. Kirkwood, J. Chem. Phys., 1935, 3, 300.

25 A. Münster, in Statistical Thermodynamics, Springer, Berlin, 1969, Vol. 1, p. 338.

26 G. Hummer, A. E. García and D. M. Soumpasis, Biophys. J., 1995, 68, 1639.

27 I. Z. Fisher and B. L. Kopeliovich, Dokl. Akad. Nauk. SSSR, 1960, 133, 81, [Sov. Phys.Dokl. 5, $761(1960)]$.

28 H. S. Ashbaugh and M. E. Paulaitis, J. Phys. Chem., in press, 1996.

29 S. Garde, G. Hummer, A. E. García, L. R. Pratt and M. E. Paulaitis, submitted, 1995.

30 W. J. McNeil, W. G. Madden, A. D. J. Haymet and S. A. Rice, J. Chem. Phys., 1983, $\mathbf{7 8}, 388$.

31 B. Bildstein and G. Kahl, Phys. Rev. E, 1993, 47, 1712. 
32 G. Hummer and D. M. Soumpasis, J. Chem. Phys., 1993, 98, 581.

33 A. D. J. Haymet, S. A. Rice and W. G. Madden, J. Chem. Phys., 1981, 75, 4696.

34 P. Attard, J. Chem. Phys., 1989, 91, 3072.

35 J.-P. Hansen and I. R. McDonald, Theory of Simple Liquids, 2nd ed., Academic Press, London, UK, 1986.

36 A. H. Narten and H. A. Levy, J. Chem. Phys., 1971, 55, 2263.

37 A. K. Soper and M. G. Phillips, Chem. Phys., 1986, 107, 47.

38 D. L. Beveridge, M. Mezei, P. K. Mehrotra, F. T. Marchese, G. Ravi-Shanker, T. Vasu and S. Swaminathan, in Molecular-Based Study of Fluids, Vol. 204 of Advances in Chemistry, ed. J. M. Haile and G. A. Mansoori, American Chemical Society, Washington, DC, 1983, pp. 297-351.

39 W. L. Jorgensen, J. Chandrasekhar, J. D. Madura, R. W. Impey and M. L. Klein, J. Chem. Phys., 1983, 79, 926.

40 A. K. Soper, Nucl. Instr. Meth. Phys. Res. A, 1995, 354, 87.

41 D. M. Soumpasis, P. Procacci and G. Corongiu, IBM DSD report, October 1991, IBM (unpublished).

42 U. Niesar, G. Corongiu, E. Clementi, G. R. Kneller and D. K. Bhattacharya, J. Phys. Chem., 1990, 94, 7949.

43 H. J. C. Berendsen, J. P. M. Postma, W. F. van Gunsteren and J. Hermans, in Intermolecular Forces: Proceedings of the 14th Jerusalem Symposium on Quantum Chemistry and Biochemistry, ed. B. Pullman, Reidel, Dordrecht, Holland, 1981, pp. 331-342.

44 O. A. Karim and A. D. J. Haymet, Chem. Phys. Lett., 1987, 138, 531.

45 O. A. Karim and A. D. J. Haymet, J. Chem. Phys., 1988, 89, 6889. 
46 O. A. Karim, P. A. Kay and A. D. J. Haymet, J. Chem. Phys., 1990, 92, 4634.

47 B. B. Laird and A. D. J. Haymet, Chem. Rev., 1992, 92, 1819.

48 W. L. Jorgensen and J. Tirado-Rives, J. Am. Chem. Soc., 1988, 110, 1657.

49 A. Wlodawer, J. Deisenhofer and R. Huber, J. Mol. Biol., 1987, 193, 145.

50 F. C. Bernstein, T. F. Koetzle, G. J. B. Williams, E. F. Meyer, Jr., M. D. Brice, J. R. Rodgers, O. Kennard, T. Shimanouchi and M. Tasumi, J. Mol. Biol., 1977, 112, 535.

51 N. C. Seeman, J. M. Rosenberg, F. L. Suddath, J. J. P. Kim and A. Rich, J. Mol. Biol., $1976,104,109$.

52 W. Saenger, Principles of Nucleic Acid Structure, Springer, Berlin, 1984.

53 E. Westhof and D. L. Beveridge, in Water Science Reviews, ed. F. Franks, Cambridge Univ. Press, Cambridge, UK, 1990, Vol. 5, pp. 24-136.

54 E. Liepinsh, G. Otting and K. Wüthrich, Nucleic Acids Res., 1992, 20, 6549.

55 M. G. Kubinec and D. E. Wemmer, J. Am. Chem. Soc., 1992, 114, 8739.

56 H. R. Drew and R. E. Dickerson, J. Mol. Biol., 1981, 151, 535.

57 M. L. Kopka, A. V. Fratini, H. R. Drew and R. E. Dickerson, J. Mol. Biol., 1983, 163, 129.

58 G. G. Privé, U. Heinemann, S. Chandrasegaran, L.-S. Kan, M. L. Kopka and R. E. Dickerson, Science, 1987, 238, 498.

59 G. G. Privé, K. Yanagi and R. E. Dickerson, J. Mol. Biol., 1991, 217, 177.

60 E. Westhof, J. Biomol. Struct. Dyn., 1987, 5, 581.

61 G. Hummer and D. M. Soumpasis, in Structural Biology: The State of the Art; Proceedings of the Eighth Conversations in the Discipline Biomolecular Stereodynamics, ed. 
R. H. Sarma and M. H. Sarma, Adenine Press, Schenectady, NY, 1994, Vol. 2, pp. 273-278.

62 G. Hummer, D. M. Soumpasis and A. E. García, in Nonlinear Excitations in Biomolecules, Les Editions de Physique, ed. M. Peyrard, Springer, Berlin, 1995, pp. 83-99.

63 V. P. Chuprina, Nucleic Acids Res., 1987, 15, 293.

64 E. Liepinsh, W. Leupin and G. Otting, Nucl. Acids Res., 1994, 22, 2249.

65 J. L. Sussman, S. R. Holbrook, R. W. Warrant, G. M. Church and S.-H. Kim, J. Mol. Biol., 1978, 123, 607.

66 E. Westhof, P. Dumas and D. Moras, Biochimie, 1988, 70, 145.

67 E. Westhof, P. Dumas and D. Moras, Acta Crystallogr. A, 1988, 44, 112.

68 T. O. Fischmann, G. A. Bentley, T. N. Bhat, G. Boulot, R. A. Mariuzza, S. E. V. Phillips, D. Tello and R. J. Poljak, J. Biol. Chem., 1991, 266, 12915.

69 H. S. Green, in The Molecular Theory of Fluids, North-Holland, Amsterdam, 1952, p. 73.

70 V. Chitarra, P. M. Alzari, G. A. Bentley, T. N. Bhat, J.-L. Eiselé, A. Houdusse, J. Lescar, H. Souchon and R. J. Poljak, Proc. Natl. Acad. Sci. USA, 1993, 90, 7711.

71 B. C. Braden, H. Souchon, J.-L. Eiselé, G. A. Bentley, T. N. Bhat, J. Navaza and R. J. Poljak, J. Mol. Biol., 1994, 243, 767.

72 J. Lescar, M. Pellegrini, H. Souchon, D. Tello, R. J. Poljak, N. Peterson, M. Greene and P. M. Alzari, J. Biol. Chem., 1995, 270, 18067.

73 G. H. Cohen, S. Sheriff and D. R. Davies, to be published, 1995.

74 E. A. Padlan, E. W. Silverton, S. Sheriff, G. H. Cohen, S. J. Smith-Gill and D. R. Davies, Proc. Natl. Acad. Sci. USA, 1989, 86, 5938. 
75 F. Alary, J. Durup and Y.-H. Sanejouand, J. Phys. Chem., 1993, 97, 13864.

76 F. P. Schwarz, D. Tello, F. A. Goldbaum, R. A. Mariuzza and R. J. Poljak, Eur. J. Biochem., 1995, 228, 388.

77 D. Tello, F. A. Goldbaum, R. A. Mariuzza, X. Ysern, F. P. Schwarz and R. J. Poljak, Biochem Soc. Trans., 1993, 21, 943.

78 K. A. Hibbits, D. S. Gill and R. C. Willson, Biochem., 1994, 33, 3584.

79 K. Tsumoto, Y. Ueda, K. Maenaka, K. Watanabe, K. Ogasahara, K. Yutani and I. Kumagai, J. Biol. Chem., 1994, 269, 28777. 


\section{FIGURES}

Fig. 1 Water-oxygen triplet correction, $\Gamma(r, r, s)=g^{(3)}(r, r, s) /\left[g^{(2)}(r)\right]^{2} g^{(2)}(s)$, for (a) equilateral $(s=r)$ and (b) isosceles triangles (inset). Arrows in (b) indicate equilateral and isosceles triangles with hydrogen-bond distance. Results in (a) and (b) are from Monte Carlo simulations of 256 SPC water molecules using generalised reaction-field (GRF) electrostatics. Also shown in (a) are results of a 512 particle simulation using Ewald-summation electrostatics (dashed line).

Fig. 2 Water-oxygen density at the ice-water interface in units of the bulk-water density $\rho_{0} . d$ is the distance from the closest plane of oxygen atoms in the ice phase. $(+)$, PMF expansion including only pair correlations; ( $\square$ ), PMF expansion including pair and triplet correlations. Solid lines: explicit Monte Carlo simulations using different system sizes and methods for electrostatic interactions.

Fig. 3 Hydration of rigid model peptides from MD simulations and PMF calculations. Results for an $\alpha$-helix and a $\beta$-hairpin from BPTI are shown in panels (a) and (b), respectively. A magenta sphere is located at each grid point $(0.1 \mathrm{~nm}$ grid width) for which the MD water density $\geq 5 \rho_{0}$. PMF grid points $(0.025 \mathrm{~nm}$ grid width) of high density are labeled with cyan spheres of various sizes. The local densities are divided into classes of low density $\left(3 \leq \rho / \rho_{0}<5\right)$; medium density $\left(5 \leq \rho / \rho_{0}<7\right)$; and high density $\left(\rho / \rho_{0}>7\right)$. These grid points are illustrated by spheres of radii $0.03,0.04$ and $0.05 \mathrm{~nm}$, respectively. In panel (b), MD points of high density with no corresponding PMF density are shown in green (top left; near N31) and yellow (bottom right; near T32). 
Fig. 4 Hydration of tRNA from x-ray crystallography and PMF calculations. Results are shown for tRNA Asp [A and B form 2tra and 3tra in panels (a) and (b), respectively] and tRNA $^{\text {Phe }}[4$ tra and 6 tna in panels (c) and (d)]. Small magenta spheres show water molecules in the crystal. The smallest cyan spheres indicate grid points (on a $0.03 \mathrm{~nm}$ grid) with calculated PMF densities between 3 and $5 \rho_{0}$; larger cyan spheres indicate densities greater than $5 \rho_{0}$. Atoms of the bases, ribose and phosphate group are shown in yellow, white and red, respectively. In panels (c) and (d), water molecules of the original refinement ${ }^{65}$ are shown in blue. Crystal water of the re-refinement ${ }^{67}$ are shown in magenta. Black arrows indicate conserved water molecules, as identified by Westhof et $l^{66}{ }^{66}$

Fig. 5 Hydration of the antibody-antigen contact interface of the Fv fragment [panel (a); $1 \mathrm{vfb}^{14}$ ] and Fab fragment [panel (b); $1 \mathrm{fdl}^{68}$ ] of antibody D1.3 complexed to HEL. The lysozyme molecule has been removed for visualisation purposes, but was considered in the calculations. Yellow and white colours are used for heavy and light chain. Crystal water molecules are shown in magenta. PMF grid points with high water density are shown in cyan (see also Fig. 4). No crystal water was reported in the PDB file $1 \mathrm{fdl}$.

Fig. 6 Hydration of the photosynthetic reaction centre. ${ }^{13}$ Panel (a) shows the hydration between the special pair (yellow) and the nearest haem (red). Panel (b) shows the water channel near the ubiquinone $\mathrm{Q}_{B}$. Protein atoms are shown in gray, with parts of the protein removed to visualise the hydration of the protein interior. Crystal water is shown in magenta; PMF high-density grid points are shown in cyan (see also Fig. 4).

Fig. 7 Measured antibody-antigen binding entropies versus estimated entropy of dehydration of polar groups. Thermodynamic results are shown for antibodies D1.3 ${ }^{76}$ (diamond), F9.13. $7^{76}$ (open square; HEL data), D44.1 ${ }^{76}$ (star), D11.15 $5^{77}$ (open triangle), HyHel-5 ${ }^{78}$ (full square) and HyHel-10 $0^{79}$ (full triangle). The solid line is a linear fit. 
অ্ড

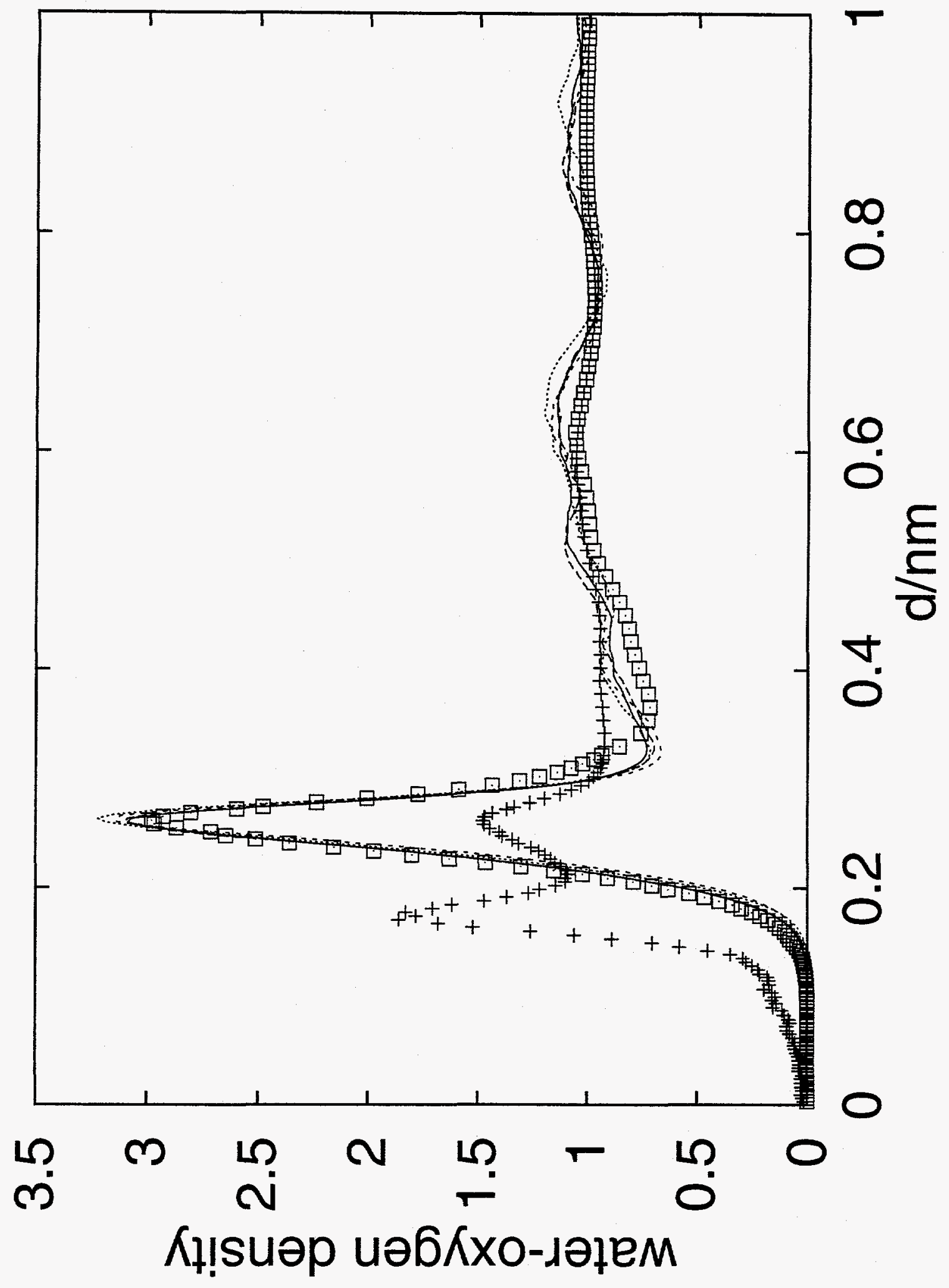




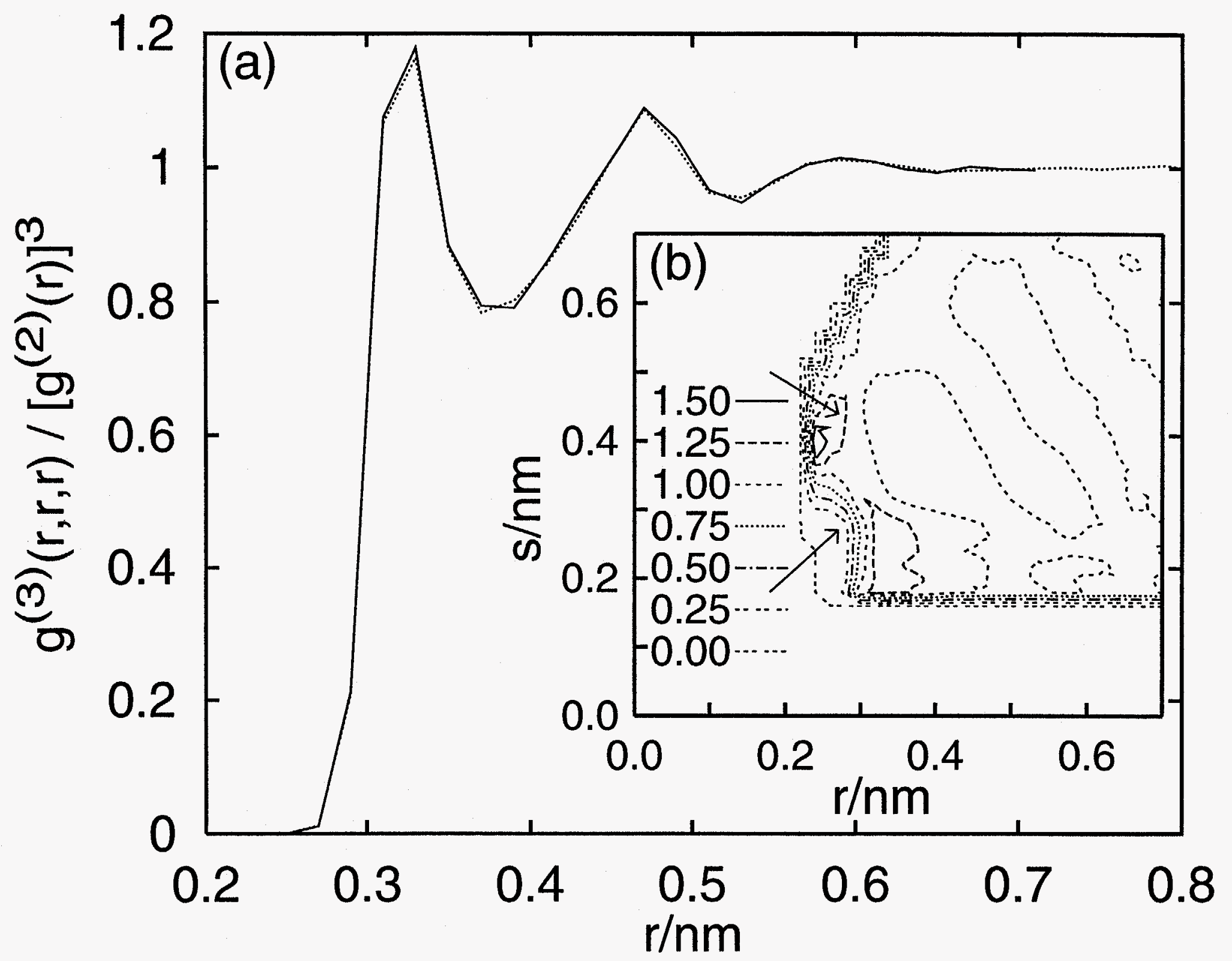

$F_{1<2}$ 


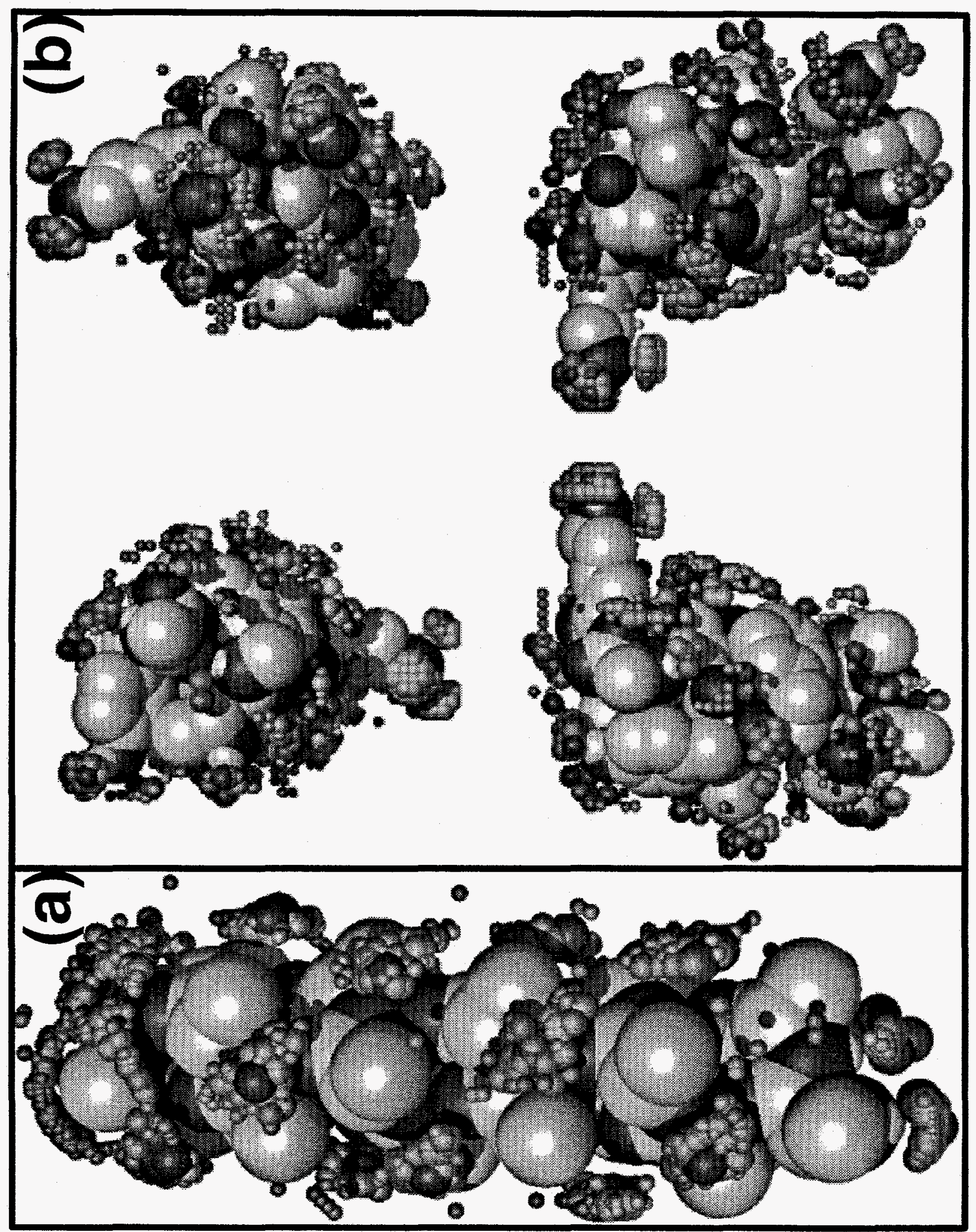




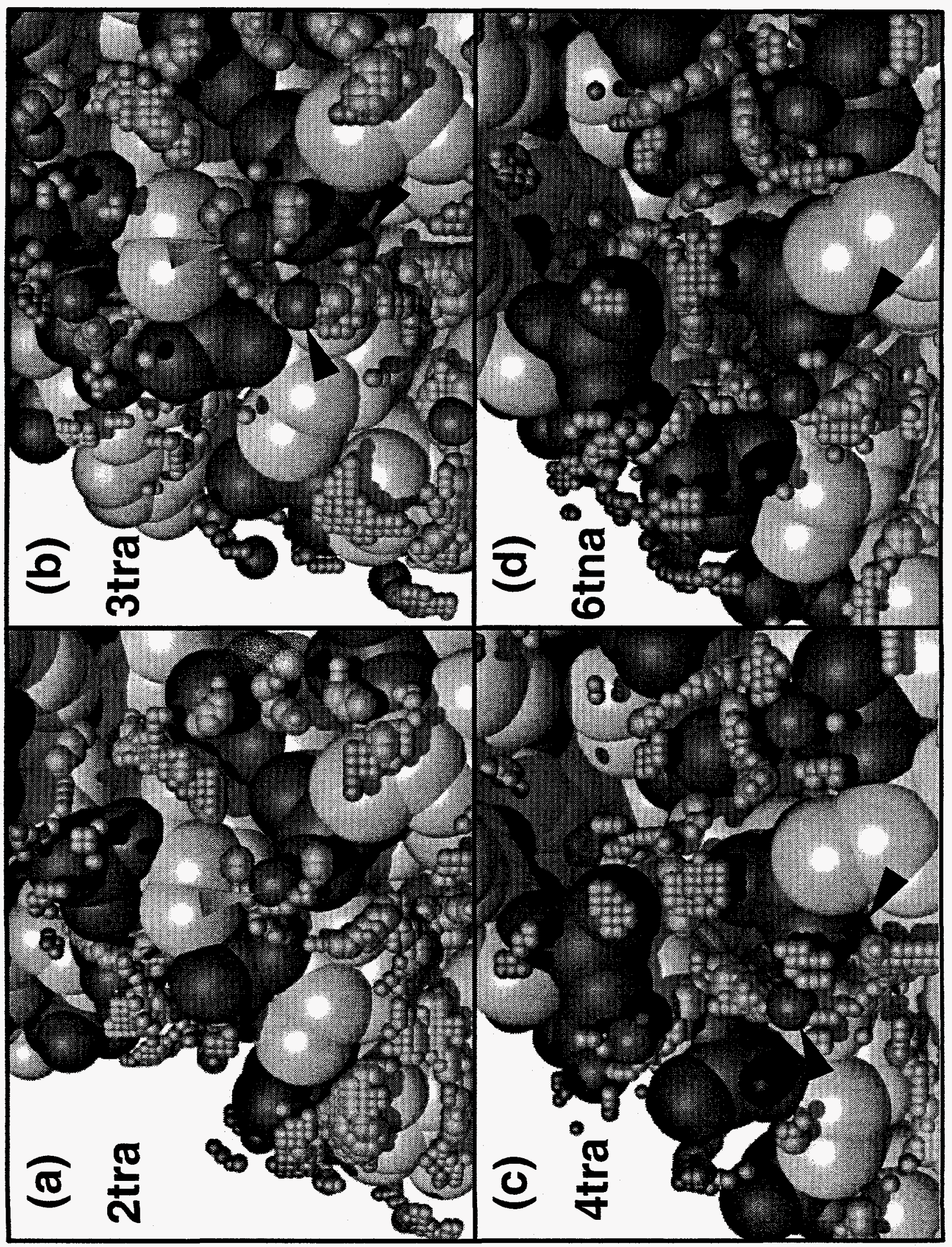




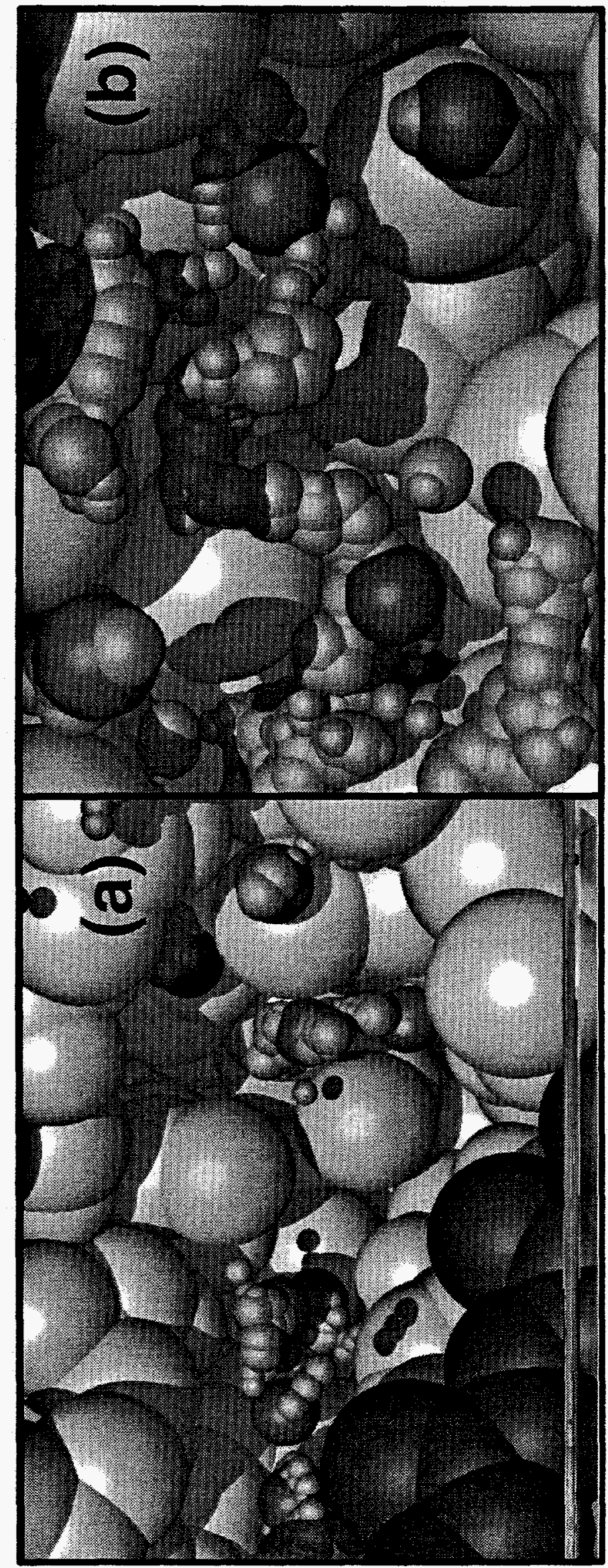




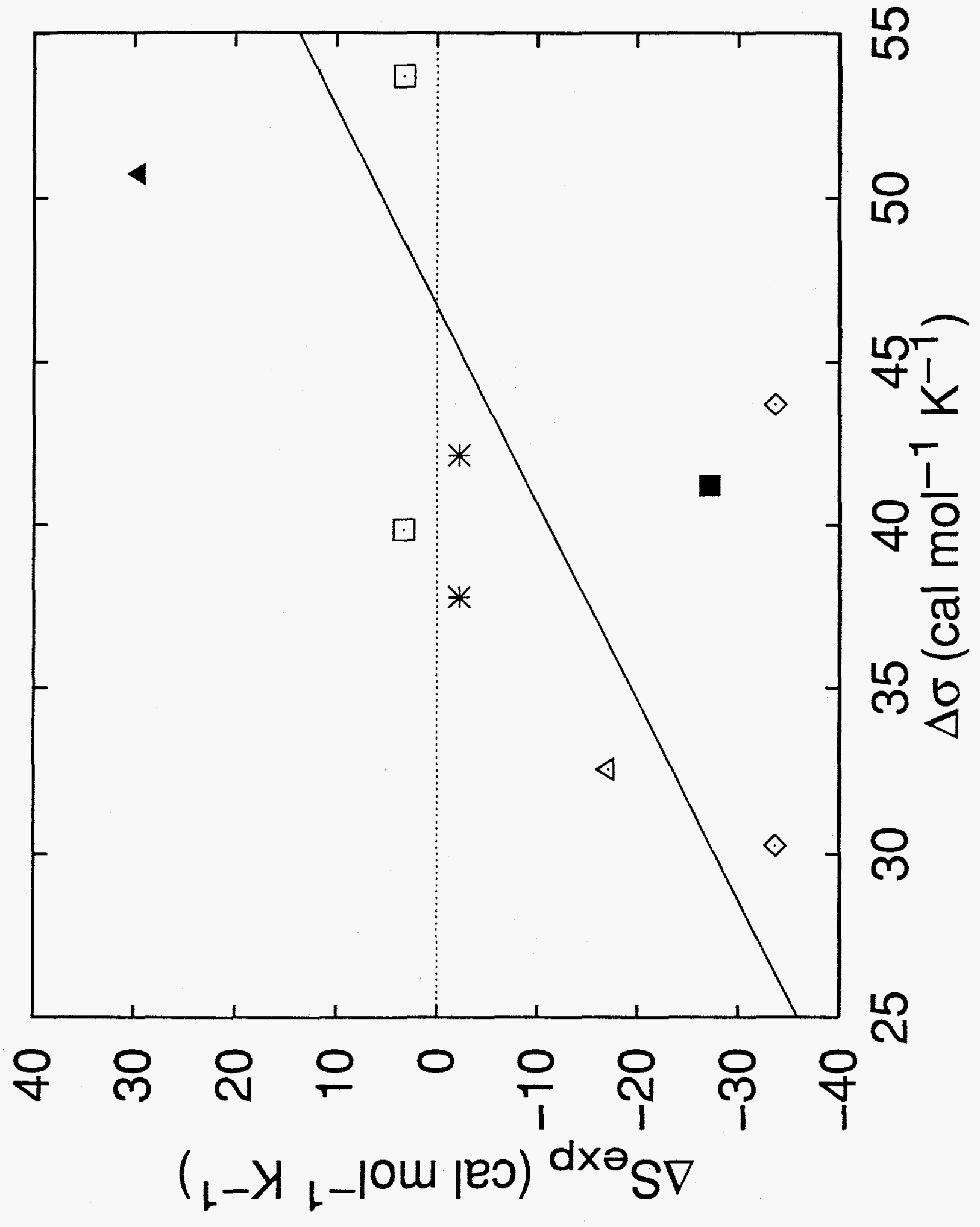

